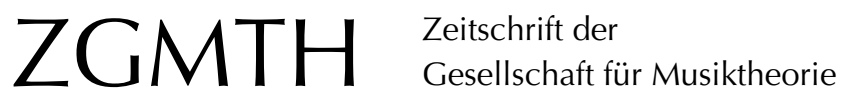

Jeßulat, Ariane (2007): Harmonische Systeme des ausgehenden 19. Jahrhunderts und ihre Anwendung auf die Musik Richard Wagners. ZGMTH 4/3, 261-273.

https://doi.org/10.31751/260

(C) 2007 Ariane Jeßulat

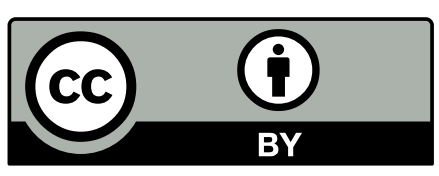

Dieser Text erscheint im Open Access und ist lizenziert unter einer Creative Commons Namensnennung 4.0 International Lizenz.

This is an open access article licensed under a Creative Commons Attribution 4.0 International License.

veröffentlicht / first published: 01/07/2007

zuletzt geändert / last updated: 07/04/2009 


\title{
Harmonische Systeme des ausgehenden 19. Jahrhunderts und ihre Anwendung auf die Musik Richard Wagners ${ }^{1}$
}

\author{
Ariane Jeßulat
}

Die großen systematischen Entwürfe zu Harmonik und Tonalität, deren Wurzeln überwiegend in der Mitte des 19. Jahrhunderts liegen und deren Ausläufer heute noch in einer vielfach modifizierten `Funktionstheorie` in Gebrauch sind, stehen wegen ihres verfehlten Anspruchs, auf historisch unabhängigen, musikalischen Gesetzmäßigkeiten zu beruhen, in der Kritik. Andererseits aber war es eben diese Kritik, insbesondere jene zu Beginn des 20. Jahrhunderts, welche überhaupt erst das Potential freilegte, das die Vorstellung einer harmonischen `Funktion` von der Fundamentschritt-Theorie Rameaus unterscheidet. Ebenso deutlich wird, dass eine vermeintlich spekulative Grundannahme, wie beispielsweise die einer Symmetrie zwischen Dominante und Subdominante, nicht das Ergebnis naturwissenschaftlich messbarer Tonbeziehungen ist, sondern ein ästhetisches Postulat, das auf die Musik der Zeit reagiert. Die sromantische Harmonikı, besonders scharf artikuliert in den Musikdramen Richard Wagners, wirkt dabei zu gleichen Teilen als Vorbild und Indikator.

In der Mitte des 19. Jahrhunderts zeichnete sich ein grundlegender Wandel in der Konzeption musiktheoretischer Lehrwerke ab. Waren die Inhalte traditioneller Generalbass-, Kompositions- oder Harmonielehren in der ersten Hälfte des 19. Jahrhunderts noch für eine direkte Anwendung im Generalbassspiel, in der musikalischen Interpretation oder in der Komposition gedacht, so setzt Moritz Hauptmanns 1853 erschienene Publikation Die Natur der Harmonik und der Metrik in Intention und Darstellung einen neuen Akzent: Die Nähe zu den exakten Wissenschaften wird gesucht, die Verbindung zur musikalischen Praxis gemieden. Es geht nicht um den Erwerb musikalischer Fähigkeiten; vielmehr soll der bereits ausgebildete Musiker die Gesetze musikalischer Schönheit auf logischem Wege erkennen und ableiten können. Hauptmann ist darin so konsequent, dass er im gesamten Buch auf Notenbeispiele verzichtet. ${ }^{2}$

Hugo Riemann, der in seinen frühen Veröffentlichungen Hauptmanns Thesen in weiten Teilen lediglich referierte, löste dessen Denkmodelle in einem groß angelegten Miss-

1 Dieser Beitrag wurde in einer ersten Fassung im Rahmen der »Fachtagung Musiktheorie und Hörerziehung - Unterrichtsangebote für Musikpädagogen« gehalten, die vom 10. bis zum 11. März 2007 in Kooperation mit der GMTH an der Landesmusikakademie in Sondershausen stattfand.

2 Zur erkenntnistheoretischen Qualität des in Hauptmanns Schriften dargestellten Ansatzes vgl. Rummenhöller 1966, 11-38. 
verständnis ihrer ursprünglichen Intention aus ihrer erkenntnistheoretischen Hermetik und arbeitete auf dieser Grundlage im Laufe von 40 Jahren jene Lehre aus, die man heute als `Funktionstheorie bezeichnet. Riemanns Bestreben, Anschluss an die Naturwissenschaften und die Philosophie zu finden, ist charakteristisch für einen großen Teil des musiktheoretischen Schrifttums der zweiten Hälfte des 19. Jahrhunderts. So erwartete er von der Akustik Beweise für sein System, das alle musikalischen Gesetze aus dem reinen Dur- oder Molldreiklang abgeleitet hatte. ${ }^{3}$ Und obwohl die Errichtung harmonischer Systeme nach Prinzipien der deduktiven und daher allgemeingültigen Logik noch im 19. Jahrhundert durch naturwissenschaftliche, historische und ethnologische Erkenntnisse in Frage gestellt wurde ${ }^{4}$, konnte sich die Vorstellung einer auf ästhetischen Naturgesetzen` aufbauenden ımusikalischen Logikı etablieren.

Eine kritische Reaktion auf die abstrakte, deduktive Harmonielehre stellen manche musiktheoretische Publikationen zu Beginn des 20. Jahrhunderts dar, wobei die Arbeiten Arnold Schönbergs (1911), Heinrich Schenkers (1906) und Ernst Kurths (1920) durch ihre Qualität und Bedeutung herausragen. Die Gesetze der Dur-Moll-Tonalität als überhistorisch zu betrachten, hatte sich endgültig als unhaltbar herausgestellt, und die Demonstration sgrundlegender Gesetze an nackten, mehr oder weniger unrhythmisierten Akkorden leistete der ohnehin vorherrschenden Tendenz in der musikalischen Anfangslehre Vorschub, abstraktes und praktisch angewandtes Denken ohne stilistischen Halt durch den musikalischen Zusammenhang in Übungssätzen miteinander zu vermischen, die der reinen Demonstration des Regelwerks dienen sollten. Nach Vorstellung einer überholten Hermeneutik wurde mit diesen abstrakten Tonsätzen der Anspruch erhoben, musikalische Bausteine zu liefern, aus denen sich die musikalische Sprache zusammensetzen lassen sollte. Sogar Schönberg, der im Vorwort zur Harmonielehre denjenigen Unterricht verurteilt, der an Stelle des lebendigen Vorbilds das scheinbar sichere, aber primitive und letztlich die künstlerische Entwicklung hemmende harmonische System setzt ${ }^{5}$, mutet dem Schüler im Sinne eines falsch verstandenen Handwerks das Studium unzähliger isolierter, unrhythmisierter und zum großen Teil satztechnischer fragwürdiger Notenbeispiele zu. Der heuristische Impuls dieses Buches, tonale Zusammenhänge aus

3 »Mit Spannung erwarte ich nun - und mit mir alle ernsten Interessenten der endlichen Herstellung eines wirklich widerspruchslosen Kontakts der Theorie der Tonempfindungen mit der praktischen Kunstübung - den unentbehrlichen Ausbau der Verschmelzungstheorie Stumpfs, welche uns den Begriff der Harmonie, des konsonanten Akkords bringt." (Riemann 1901b, 43)

4 So geht Hauptmann selbstverständlich davon aus, das von ihm entwickelte Regelwerk sei stilübergreifend auf alle Musik anzuwenden: „Der musikalische Fehler ist ein logischer Fehler, ein Fehler für den allgemeinen Menschensinn, nicht für einen musikalischen Sinn insbesondere. Die Regeln des musikalischen Satzes auf ihre wesentliche Bedeutung zurückgeführt, sind nur die Regeln für das genuin Verständliche überhaupt und sind in dieser Bedeutung für einen Jeden zu fassen, da sie nur Allbekanntes in ihm ansprechen.« $(1853,7)$ Zur Zeit der Abfassung dieses Satzes bestanden kaum Gründe, an der Existenz eines »für alle Musik« gültigen, deduktiven Regelwerks zu zweifeln.

5 »Und hier haben wir den wundesten Punkt der Theoretiker angestoßen: ihre Theorien wollen als praktische Ästhetik wirken; wollen den Schönheitssinn beeinflussen, daß er beispielsweise durch Harmoniefolgen solche Wirkungen hervorbringe, die für schön angesehen werden können; wollen das Recht haben, solche Klänge und Folgen auszuschließen, die für unschön gelten. Aber diese Theorien sind nicht so gebaut, daß aus ihren Grundsätzen aus der folgerichtigen Weiterbildung dieser Grundsätze die ästhetische Bewertung sich von selbst ergibt! (Schönberg 1911, 4) 
vorher kaum gekannter analytischer Distanz heraus zu verstehen und darzustellen, wird durch diese Rückstände einer poetischen Elementarlehre nicht unterstützt.

Ebenso ist auch die bei Schenker, Kurth und Schönberg in den Vordergrund tretende Werkanalyse nicht frei von abstrakten harmonischen Denkmodellen des 19. Jahrhunderts: Da bei jeder analytischen Tätigkeit theoretische Grundannahmen eine erkenntnisleitende Funktion besitzen, kommt Riemannsches bzw. Hauptmannsches Gedankengut durchaus zur Anwendung, wenn auch nicht in der Unbedingtheit abstrakt formulierter Thesen. Allerdings zeigt sich, dass alle drei Autoren bereits im Bewusstsein einer historischen Distanz gegenüber dem Phänomen der Dur-Moll-Tonalität argumentieren, wenn auch auf jeweils unterschiedliche Art: Schönberg beschreibt das Wesen der Tonalität, indem er sie - salopp gesagt - an ihren eigenen Gesetzen zugrundegehen lässt. Kurth wendet sich aus der Erfahrung der neueren Musik des frühen 20. Jahrhunderts heraus der Musik Wagners zu, die für ihn einen Prüfstein tonaler Gesetze darstellt. Schenker schließlich legt bereits in seiner Harmonielehre einen Zeitraum zwischen Bach und Wagner fest, für den die von ihm dargelegten Gesetze gelten, alle andere Musik wertet er ästhetisch im Sinne eines snoch nicht oder snicht mehr entschieden ab.

Die im skizzierten Zeitraum entwickelten Vorstellungen über die Logik harmonischer Gesetze könnten zum Teil verschiedener nicht sein, doch haben sie eine grundlegende Gemeinsamkeit: Sie behaupten spezifische, die Harmonik betreffende Symmetrien: den >Dur-Moll-Dualismus` und das Gegensatzpaar Dominante und Subdominante.

Ich möchte mich im Folgenden auf Beobachtungen zum Gegensatzpaar Dominante und Subdominante beschränken und zeigen, dass sich hiermit ein ästhetisches Postulat verbindet und nicht eine Gesetzmäßigkeit, die a priori in der Natur tonaler Musik läge. Die Tatsache allerdings, dass in der zweiten Hälfte des 19. Jahrhunderts, besonders in der Musik Richard Wagners, harmonische Symmetrien wie diese zum Teil schärfer und pointierter komponiert wurden als zuvor, mag mit dazu beigetragen haben, dass der zeitgenössischen Musiktheorie zufolge die Dur-Moll-Tonalität auf diversen Dualismen beruht. Auch die wissenschaftlich ausgerichtete Harmonielehre des 19. Jahrhunderts ist trotz ihrer Anklammerung an abstrakte Regeln und Notenbeispiele durchaus nicht ästhetisch neutral: Sie ist ein Reflex auf die Musik ihrer Zeit.

Ernst Kurth, der grundsätzlich Riemanns Lehre nicht anzweifelt, äußert sich über die Dynamik harmonischer Vorgänge folgendermaßen:

Schon die harmonischen Grundvorgänge, die dominantisch-subdominantischen Wirkungen, sind rein energetisch begründet, ebenso wie bereits die schlichtesten konsonanten Akkordgebilde, Dur- und Molldreiklang nur als Gegensatzformen potentieller Energie Grundformen einer zweifach und gegensätzlich ausstrahlenden Harmonieentwicklung der beiden Tongeschlechter darstellen. ${ }^{6}$ 
Kurths Äußerung steht für den Wandel von der interpunktischen Kadenzlehre des 18. Jahrhunderts zur Lehre harmonischer Funktionen des 19. Jahrhunderts, in der Stimmführung und das Verhältnis von Konsonanz und Dissonanz, Kategorien, die z. B. bei Rameau und seinen direkten Nachfolgern bis hin zu Sechter von zentraler Bedeutung für das Verständnis von Tonart sind, keine wesentliche Rolle mehr spielen.

An den beiden folgenden, zwei sromantischen Werken entnommenen Inszenierungen einer sogenannten `Grundkadenz wird das Gemeinte spürbar:

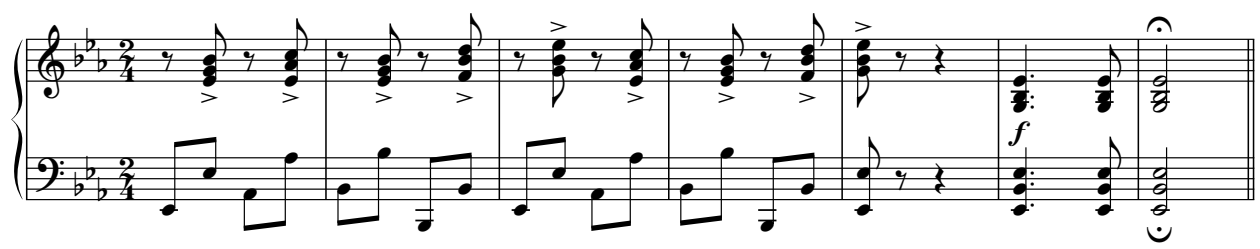

Beispiel 1: Robert Schumann, Dichterliebe op. 48, Nr. 11 »Ein Jüngling liebt ein Mädchen«, Schluss

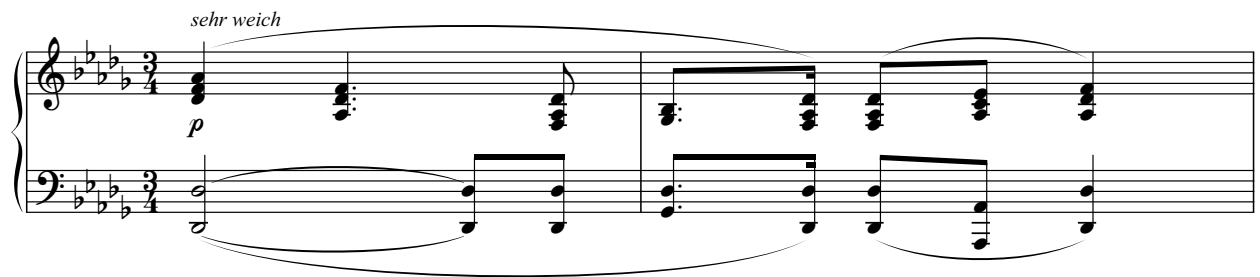

Beispiel 2: Richard Wagner, Das Rheingold, 1. Aufzug, 2. Szene

Bei Schumann handelt es sich um die offenkundig ironische Verwendung einer Schulkadenz, die für die »alte Geschichte« aus Heines Gedicht steht. Das Modell hat im Lied Veränderungen und Alterationen erfahren, und läuft sich nun - auf Grundakkorde reduziert - im Klaviernachspiel tot. Auch bei Wagner könnte eine ironische Konnotation der salten Ordnung، intendiert sein, die in der Erzählhandlung durch den Bau des Schlosses Walhall repräsentiert wird, in der musikalischen Handlung durch Motive wie das sogenannte sWallhall-Motiv in Klang gesetzt erscheint.

Der Vergleich macht aber eine Tendenz spürbar: Trotz der Parallelität beider Beispiele hat die Subdominante bei Wagner mehr Gewicht als bei Schumann. Sie erklingt als Wendepunkt und Hauptakzent des Motivs, wohingegen der Akkord der Dominante sehr leicht ist. Er dient lediglich der Wiederherstellung der Tonika. Die dabei zwischen den Oberstimmen entstehenden Hornquinten prägen die Klanglichkeit der Fortschreitung mehr als der Akkordwechsel im Rahmen der authentischen Kadenz V-I. So gesehen scheint das Walhall-Motiv nahezu eine Verkörperung des von Riemann aufgestellten Axioms zu sein, dem zufolge die erste Tonika der These entspricht, der die Subdominante als Antithese gegenübergestellt wird, und die Verbindung von Dominante und 
Tonika als Synthese zu gelten hat.7 Nun wirkt das Walhall-Motiv aber ganz und gar nicht snatürlichı, sondern, ähnlich wie das Beispiel bei Schumann, durch seinen Verzicht auf Dissonanzen äußerst artifiziell.

Noch im 17. und frühen 18. Jahrhundert hat die IV. Stufe längst nicht diesen Stellenwert, geschweige denn einen Charakter, der sie als `Gegensatz ‘ zur V. Stufe verstehen ließe. Auch ihre formbildende Kraft ist vergleichsweise gering. So kommt sie im Kadenzplan eines Präludiums der Corelli-Zeit meist gar nicht vor. Noch die aus Bachs Generalbassunterricht dokumentierten einfachen Spielstücke haben Ausweichungen lediglich zur V. und VI. Stufe. Bei den Modi weisen nur 4. und 8. Ton die IV. Stufe als Ziel einer Sekundärklausel auf.

Zwar haben `Quintentürme` bereits in Kompositionen des 15. Jahrhunderts im Dienste musikalischer Expressivität Verwendung gefunden:
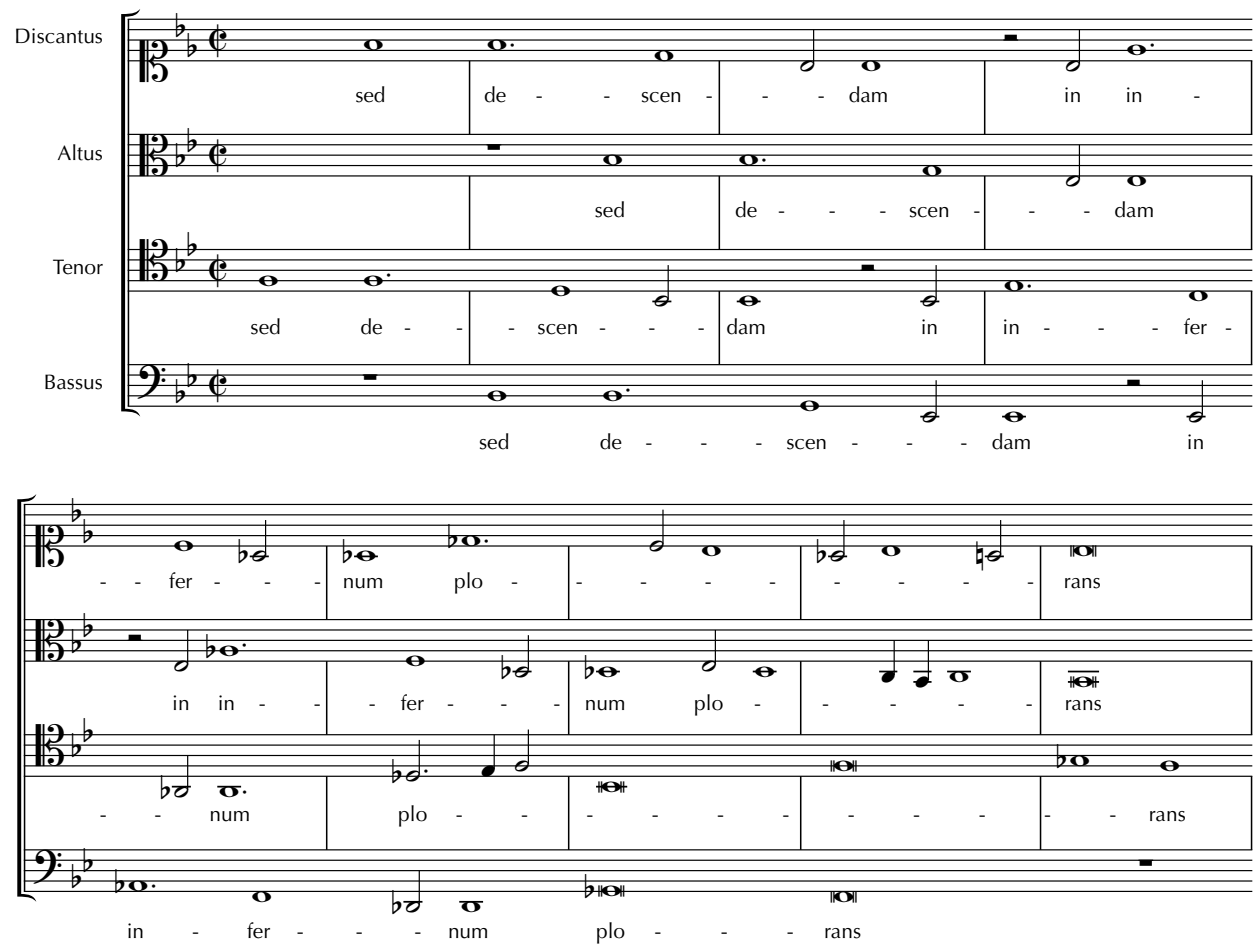

Beispiel 3: Josquin Desprez, Absalom fili mi, Mensur 60-77

Ebenso gibt es Formen der `Regola dell'Ottavas, in denen die Tonart vor allem durch die Dreiklänge der I., IV. und V. Stufe zum Ausdruck gebracht wird:

7 Riemann 1901a, 3 und Hauptmann 1853, 25, § $18 \mathrm{ff}$. 


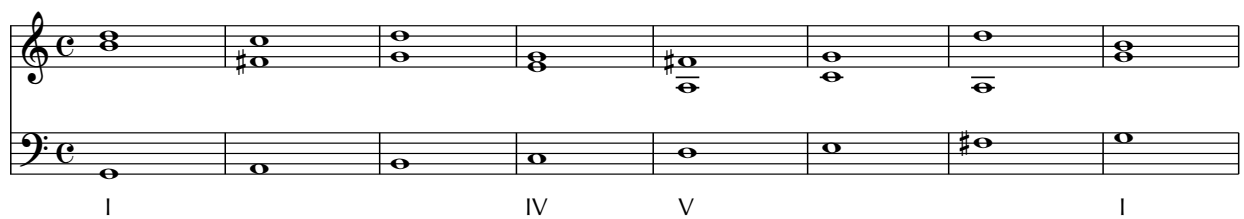

Beispiel 4: Antonio Bruschi, Regole per il contrapunto e per l'accompagnatura (1711) ${ }^{8}$

Dennoch setzt die Vorstellung, die Tonart bestünde aus dem spannungsvollen Gleichgewicht zwischen dominantischen und subdominantischen Entitäten, eine kategoriale Aufwertung der Subdominante voraus. Möglicherweise ist der hohe Stellenwert der Subdominante in Rameaus Nouveau systême von 1726 ein Reflex auf den allmählichen Beginn dieser Entwicklung, die schließlich in den harmonischen Verhältnissen des klassischen Periodenbaus zu voller Entfaltung kommt. Damit aber ist der Prozess noch keineswegs abgeschlossen. Kurth formuliert:

Die harmonische Kadenzierung vollzieht sich statt in einfachen in potenzierten dominantischen und subdominantischen Spannungen. [...] hob z. B. eine [Doppeldominante] vor der Dominante bis in den zweiten Grad dominantischer Entwicklung [...], so war der Anstoß gegeben, diese Verhältnisse nicht nur zu weiten, sondern auch nach der entgegengesetzten Richtung zu übertragen und auch subdominantische Stufen höheren Grades eindringen zu lassen. ${ }^{9}$

Mime, der alte, gelehrte und boshafte Zwerg aus dem Ring des Nibelungen bringt in der abschließenden Kadenz seines sogenannten Erziehungsliedes zunächst eine Subdominante auf der tiefalterierten II. Stufe. Es scheint, als ließe er sich in seinem Zorn dazu hinreißen, der Passage noch mehr Emphase zu verleihen, als ihr auf dem Hintergrund der rhetorischen Tradition in der Form des $>$ Neapolitaners $\iota^{10}$ bereits ohnehin zukommt, und so folgt noch ein weiterer Quintfall zur Ces-Stufe als Subdominante in Ges-Dur. Als vermittelndes Moment findet der Ton $f$ Verwendung, der im Ges-Dur-Dreiklang als Septime, im Ces-Dur-Dreiklang als Quartvorhalt erklingt (Beispiel 6).

Wichtig in diesem Zusammenhang ist, dass Mime in Wagners Darstellung eine Art sSprachfehler hat: Als folgte seine musikalische Diktion einer Tendenz zu einem karikierten Phrygisch, singt er in f-Moll grundsätzlich ges statt $g$. So enthält seine Partie des Siegfried bis zu diesem Punkt fast ausschließlich ıneapolitanische ‘ oder eben sphrygische` II. Stufen, so dass eine Steigerung nötig scheint, um dieser Subdominante überhaupt Nachdruck zu verleihen.

Ein Vergleich mit einer auf ähnliche Weise durch ihre eigene Unterquinte gesteigerten Subdominante bei Chopin zeigt die Radikalität Wagnerscher Harmonik. Auch bei Chopin ist es die Schlusskadenz, die zunächst in einen neapolitanischen Sextakkord mündet und von dort nach mehreren Anläufen eine Quinte weiter abwärts nach Heses-Dur

8 Zitiert nach: Schubert 2002, 509.

9 Kurth 1923, $137 \mathrm{ff}$.

10 Im Sinne der Stufentheorie handelt es sich um einen grundstelligen Akkord, die Funktionstheorie spricht von einem sverselbständigten Neapolitaner`, für sie ist der Sextakkord die Grundform. 

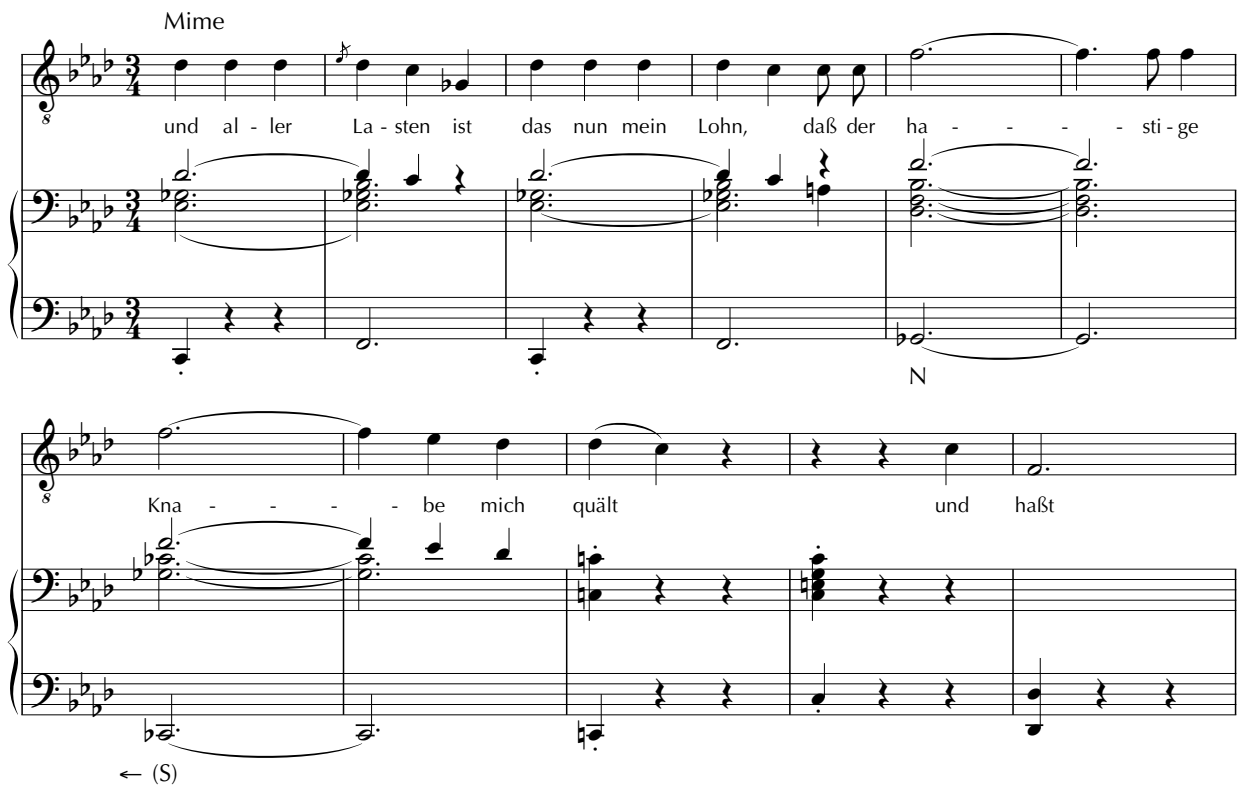

Beispiel 5: Richard Wagner, Siegfried, 1. Aufzug, 1. Szene

(das enharmonisch als A-Dur notiert erscheint) kippt. Dieser Moment ist zugleich die Kulmination der im Stück vorherrschenden Tendenz zur ständigen Abwärtsmodulation. Ihr wird hier ebenso stattgegeben wie in der Schlusskadenz von Mimes Lied. Allerdings führt Chopin den potenzierten Neapolitaner als reine Wechselharmonie wieder nach Fes-Dur zurück und geht dann regulär in die Dominante von es-Moll, während Wagner mit höchst surrealer Wirkung den Ces-Dur- und den C-Dur-Dreiklang ohne jede harmonische und metrische Abfederung aufeinanderprallen lässt.
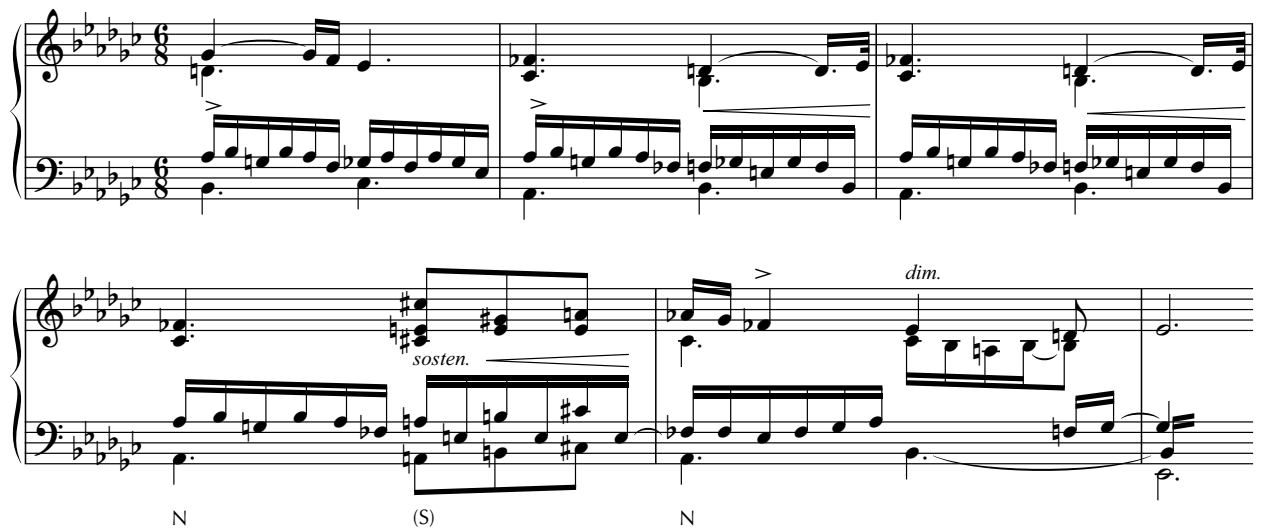

Beispiel 6: Frederic Chopin, Etüde op. 10,6 in es-Moll, T. 46-53 
In Wagners Musikdramen ist zu beobachten, dass stets wiederkehrende Motive gelegentlich einen Alterungsprozess durchlaufen. Die Emanzipation von Dissonanzen oder die Steigerung harmonischer Wirkung, die man im Allgemeinen der Musiksprache im Zuge ihres geschichtlichen Wandels unterstellt, erscheint in Wagners Musikdramen in künstlerischer Absicht reflektiert. So verläuft die 'Geschichte des Neapolitaners` ausgesprochen linear: zu Beginn der Götterdämmerung ist im Vergleich zu den Formeln im Siegfried zunächst eine weitere Steigerung, schließlich aber eine Abschwächung infolge deren inflationären Gebrauchs zu konstatieren.

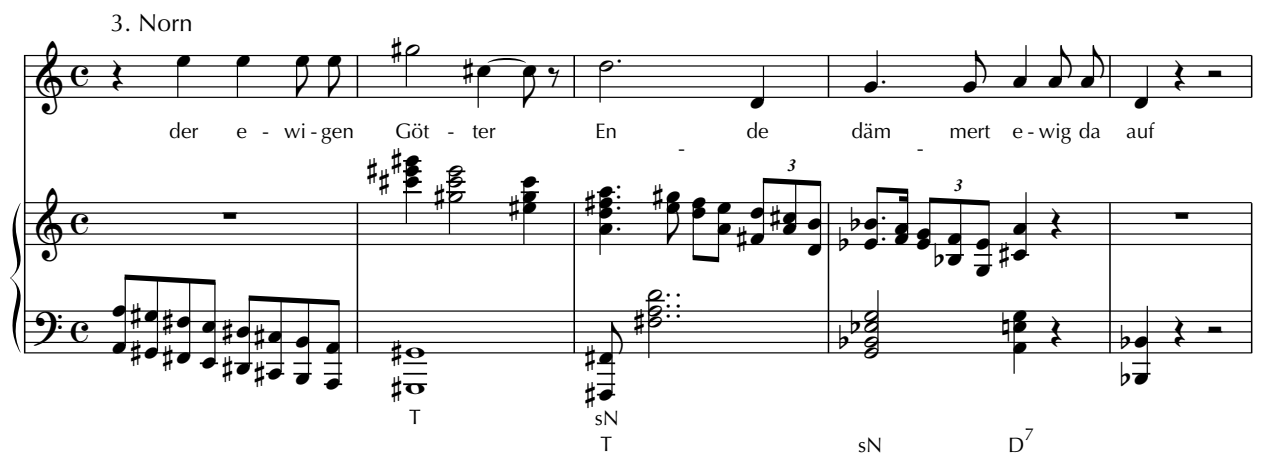

Beispiel 7: Richard Wagner, Götterdämmerung, 1. Aufzug, 1. Szene

Hier wird der neapolitanische Sextakkord von Cis-Dur zu einer Zwischentonika umgedeutet, auf die dann wiederum ein neapolitanischer Sextakkord folgt. Da Wagner diese heftige harmonische Bewegung in einen einzigen Periodennachsatz drängt, wird der Es-Dur-Sextakkord zum Textwort dämmert als doppelter Neapolitaner verstanden. Die Rede der Norn und das auf den Beginn des Walhall-Motivs folgende sogenannte `Götterdämmerungsmotivı, das immer einen neapolitanischen Sextakkord figuriert, verdeutlichen die inhaltliche Intention. Wagner hat diese Wendung zuvor in eher durchführungsartigen Passagen des Siegfried etabliert, gelegentlich auch ohne abschließende Kadenz. Dass es sich bei dem Es-Dur-Sextakkord um eine tiefalterierte II. Stufe handelt, bedarf nicht länger der kadentiellen Bestätigung, sondern ist durch den charakteristischen Klang eines unerwartet eintretenden Dur-Sextakkords verfügbar geworden. Zu Beginn der Nornenszene setzt Wagner oft Sextakkorde sfrei an, um damit - analog zum Gebrauch des Quartsextvorhalts, der als kadenzierende Dominante verstanden wird - eine Subdominante und damit eine Tonart zu markieren. Damit werden harmonische Topoi zur Formbildung herangezogen, ohne dass diese in einem konventionellen Stimmführungszusammenhang erklingen müssten.

Es überrascht nicht, dass gerade Schönberg, der in seinen theoretischen Schriften wie insbesondere der Begriff der `Fasslichkeitı anzeigt - immer wieder auf die Bedeutung der Memorierbarkeit musikalischer Strukturen für Komposition wie Rezeption hingewiesen hat, dieses Phänomen besonders plastisch beschreibt: 
Kürzung von Wendungen durch Weglassung des Wegs

Wir haben oft von der Wirkung des Klischées, der Formel gesprochen, welche dadurch charakterisiert ist, dass Wendungen, die häufig vorkommen, zu festen Gebilden von ausgesprochen eindeutigem Sinn werden. So eindeutig, dass der einmal angeschlagene Anfang sofort und automatisch die Einstellung der Erwartung auf die bestimmte Fortsetzung bewirkt: Die Formel führt zwangsläufig zu einem bestimmten Resultat. Dies vorausgesetzt, können nun die Zwischenglieder auch weggelassen, Anfang und Ende hart nebeneinandergestellt, die ganze Wendung sozusagen sgekürztı, bloß als Voraussetzung und Schluss hingesetzt werden. ${ }^{11}$

Nach Schönberg ist die „Wahrnehmung` derartiger Stellen sehr stark intellektuell vermittelt: Wer den Akkord der tiefalterierten II. Stufe nicht aus anderen musikalischen Zusammenhängen kennt und nicht um seine subdominantische Qualität weiß, wird hier lediglich eine halbtönige `Rückung`hören. Das Gefühl für harmonische Tiefe ist nicht selbstverständlich oder - vergleichbar dem sgesunden Menschenverstand renı, wie noch Hauptmann optimistisch im Vorwort seines Buches versichert hatte. ${ }^{12}$

Auch Kurth verweist auf eine harmonische Perspektive, die Alterationen oder chromatische Akkordfortschreitungen sfühlbarı macht:

Rückungswirkungen wie C-Dur/E-Dur oder C-Dur/As-Dur sind daher dynamische Gefühlsmomente, Bewegungsspannungen zur Dominante oder zur Subdominante hin. ${ }^{13}$

Noch mehr als in Schönbergs Formulierung wird deutlich, dass es sich bei der Darstellung von «Kadenzı als Funktionszusammenhang - und das muss selbstverständlich auch schon für Riemann geltend gemacht werden - keinesfalls um eine Kurzschrift für Akkorde handelt, die das Erlernen bestimmter Formeln erleichtern soll. Vielmehr ist eine räumliche Vorstellung intendiert, die auch dann noch den Erwartungshorizont des Hörers prägt, wenn die komponierte Musik den behaupteten ıldealtypus` in irgendeiner Weise anstrengt, überbietet oder in Frage stellt. Insofern bildet Wagners Musik ein klingendes Pendant zu der Akzentverschiebung von der Theorie eines 'Stimmführungszusammenhangsı zu einer Theorie funktionaler Verhältnisse zwischen `Akkorden an sichı, wie er für die Funktionstheorie maßgeblich ist. Ein solches Verständnis Wagnerscher Musik hilft analytisch insbesondere dann, wenn es um Fragen der Formbildung geht.

Bei dem vorliegenden Particell aus der Schlussszene von Tristan und Isolde (Beispiel 8) ist zunächst nicht unbedingt verständlich, was diese vom Kleinterzzirkel bestimmte Harmonik mit dem Gegensatzpaar von Dominante und Subdominante zu tun haben soll. ${ }^{14}$

11 Schönberg 1911, 432.

12 Hauptmann 1853, VI.

13 Kurth 1923, 136.

14 Die Idee eines vermittelnden Kleinterz- oder allgemeiner: Tonarten-Netzes, in dem sowohl einzelne Alterationen als auch typische Modulationswege und Sequenztypen aufgehoben sind, ist bereits bei Sechter in der Vorstellung der ‘Zwitterakkorde` angelegt und scheint sich in der österreichischen Harmonielehre (besonders bei Mayrberger) fortzusetzen. Ebenso bestehen durchaus Parallelen zu Fétis' spluritonie` und sordre omnitonique schenden Achsen-Theorien (Lendvai, Simon). Es scheint mir jedoch bei der Anwendung solcher symmetrischer Deutungsmuster sehr von Bedeutung zu sein, dass eine der Modulation übergeord- 
Isolde

As-Dur

H-Dur/Ces-Dur

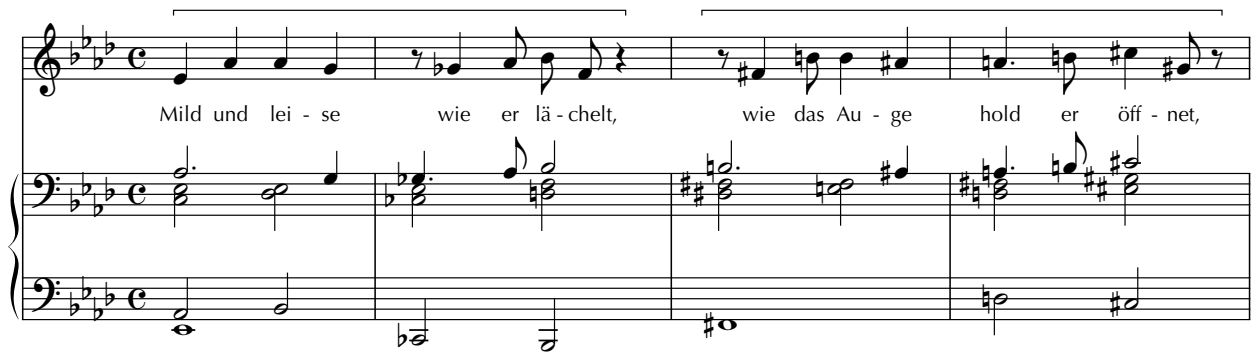

D-Dur

F-Dur

As-Dur

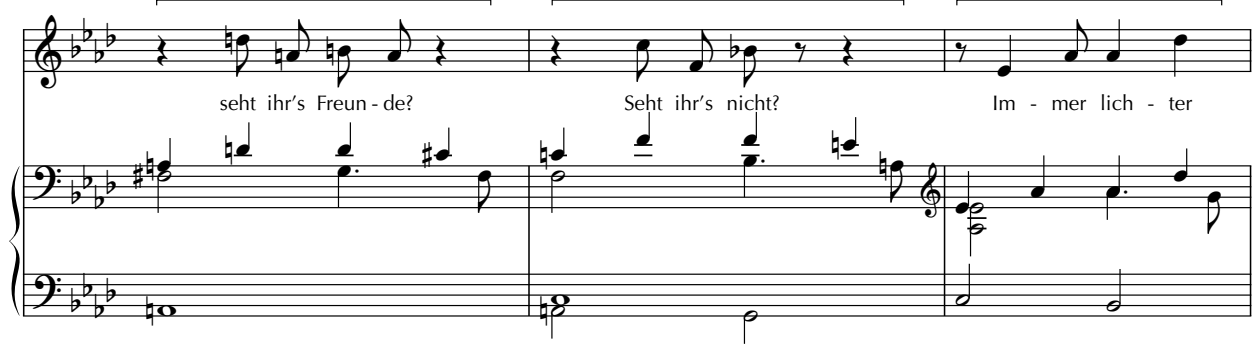

Ges-Dur

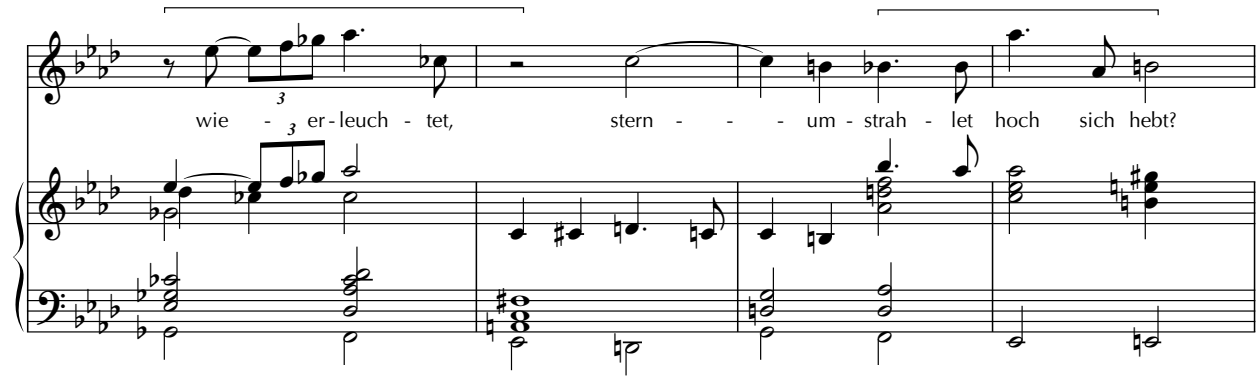

Beispiel 8: Richard Wagner, Tristan und Isolde (Schluss)

Die Ausgewogenheit dominantischer und subdominantischer Momente ist jedoch bestimmend für die Erfahrbarkeit der musikalischen Form: Im gewählten Ausschnitt kann durch die scheinbar ununterbrochene, immer dichter werdende Metamorphose eines eintaktigen Motivs hindurch die Kontur einer geschlossenen Periode in As-Dur gehört werden.

Unter dem Eindruck einer Vielzahl klassischer Perioden verwendet Schenker in seiner Harmonielehre den Begriff der ızyklischen Form`. Damit ist gemeint, dass Anfänge oder

nete intervallische Symmetrie in komponierter Musik bis in die erste Hälfte des 20. Jahrhunderts hinein kaum alleinige Grundlage der harmonischen Form, sondern eher ein Spannungshorizont ist, der zwar als Abstraktion von Durchführungs- und Introduktionsmodellen tonaler Musik entnommen, in dieser symmetrischen Konsequenz jedoch gegen diejenigen formalen und harmonischen Topoi aufgerichtet wird, die - einem Inertialsystem dur-moll-tonaler oder auch älterer Gesten zugehörig - asymmetrische Schwerpunkte setzen (vgl. dazu auch Haas 2004, 11-19, Gárdonyi 2002, 194-218 sowie Gárdonyi 1969, 169 ff.). 
Vordersätze gern dominantisch, alteriert oder anderweitig verfremdet gestaltet werden, wohingegen Nachsätze zur Tonika zurückführen. ${ }^{15}$ Die dramaturgische Qualität einer Periode aus Beethovens Streichquartett op. 95 beschreibt Schenker als »Warten auf die Subdominante $\ll .{ }^{16}$ Der Gedanke kongruiert erstaunlich mit Riemanns Modell einer Periode, deren Vordersatz vorbereitend, deren Nachsatz aber erfüllend ist. ${ }^{17}$ Im Rahmen dieser Überlegung gewinnt die Eröffnung des Kadenzvorgangs im Nachsatz besondere Bedeutung. Beispiele wie der Anfang des langsamen Satzes aus Beethovens Sonate op. 2,2 zeigen, dass dieser Moment oftmals mit einer Subdominante als Höhepunkt einhergeht.
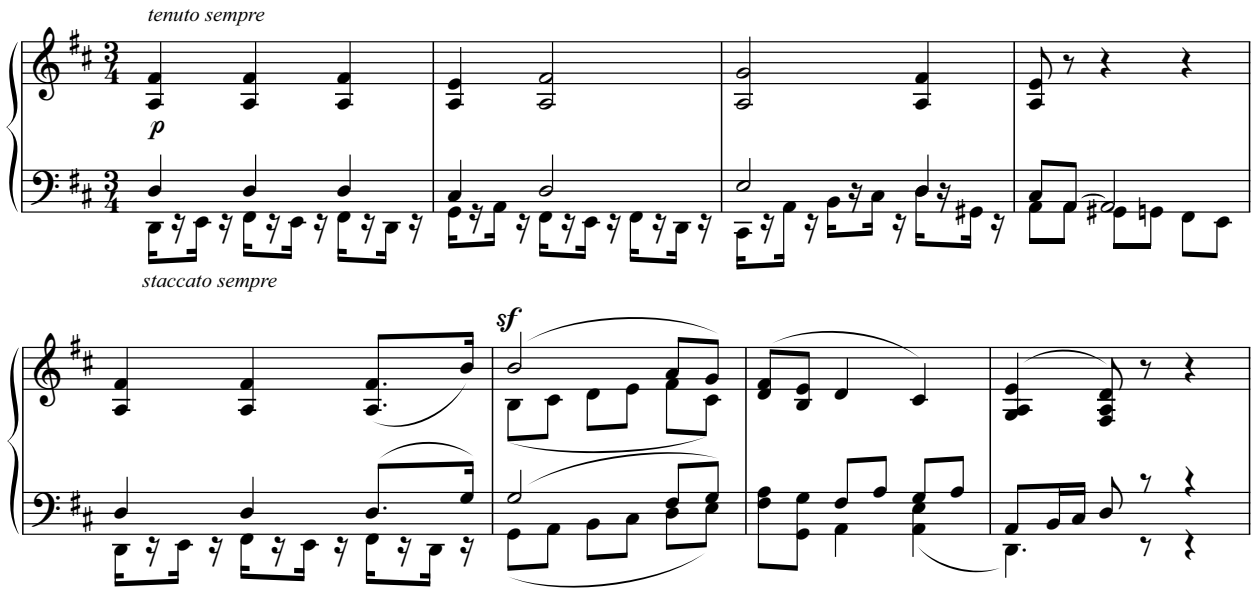

Beispiel 9: Ludwig van Beethoven, Klaviersonate A-Dur op. 2,2, II. Satz, T. 1-8

Vergegenwärtigt man sich dieses in der Literatur zur musikalischen Formenlehre oft zitierte Beispiel als Idealtypus von Harmonik, Korrespondenz und metrischen Schwerpunkten beim Hören der Wagnerschen Periode, so wird deutlich, dass die Konturen durchaus noch dieselben sind: Wagners Vordersatz setzt sich aus einer Folge von kurzen Kadenzen im Kleinterzabstand zusammen, die alle im weiteren Sinne dominantisch bzw. doppeldominantisch enden. Das Modell eines Vordersatzes wird dabei zunächst auf zwei Takte bzw. einen Takt reduziert und im Anschluss mittels des Kleinterzzirkels, der der `Auskomponierung` der Tonart As-Dur dient, wiederum vergrößert. Angesichts dieses harmonischen Reichtums auf dominantischer Ebene fehlt eine sechte`, syntaktisch in der Kadenz verankerte Subdominante. ${ }^{18}$

15 Schenker 1906, 374, § 158 »Chromatik im Dienste der zyklischen Form«.

16 Ebd., 319-325.

17 Riemann 1916, 1-21.

18 Entgegen der `Schreibweisenenharmonikı läuft der Kleinterzzirkel zyklisch abwärts (As-Ces-Eses usw.), so dass eine zyklische Abwärtsspirale die aufsteigende Sequenz transzendiert. Als dynamisches Moment gibt es demnach im Vordersatz durchaus Subdominantisches: in dieser Aufgehobenheit auch Metapher des »Ertrinkens in des Weltatems wehendem All«. 
Der Nachsatz der Periode beginnt in Takt 7 des Notenbeispiels. Die Ähnlichkeit dieses Taktes mit dem Vordersatzbeginn, das dominantische Ende von Takt 6 und nicht zuletzt die Interpunktion des Textes machen dies deutlich. Allerdings wird der durch die anhaltende Beschleunigung der Sequenz schon nach einem Takt anschließende Ces-Dur-Dreiklang in Takt 8 (entspricht Takt 6 des achttaktigen Idealtypus) nicht tonikal wie im Vordersatz, sondern als Subdominante in Ges-Dur, der Doppelsubdominante in As-Dur, weitergeführt. Der Ges Dur Dreiklang selbst erklingt nicht; seiner indirekten Darstellung durch Subdominante und Dominante verdanken sich Emphase und Raumwirkung dieser Stelle.

In dem Moment, in dem die Regelmäßigkeit des Kleinterzzirkels durch einen traditionellen, nicht alterierten Kadenzschritt IV-V unterbrochen wird, zeitlich nah genug an der Tonika As-Dur, um dem Hörer den gesamten Vorgang verständlich zu machen, tritt am melodischen Höhepunkt der Phrase ein potenzierter subdominantischer Moment ein. ${ }^{19}$ Gleichzeitig korrespondiert diese Stelle mit dem zweiten Periodentakt durch einen übergeordneten Dur-Moll-Parallelismus. Statt des erwarteten es-Moll bei »wie er lächelt» erklingt die parallele Durtonart Ges-Dur bei »wie erleuchtet« - ein Beispiel dafür, wie eng Text und Harmonie in Wagners Periodenbau zusammengehen können.

Sicherlich, man kann die Harmoniefolgen in Isoldes Liebestod relativ problemlos durch ein Stimmführungsmodell (`Consecutiva per quintam ad sextam`) erklären, das Dreiklänge im Terzabstand miteinander auf engstem Wege verbindet. Jedoch geht es nicht allein darum, Akkordfolgen zu erklären, sondern eine übergeordnete Dynamik nachzuvollziehen, die Akkordfolgen zu Perioden oder anderen größeren Formen zusammenfasst. Ähnlich wie die Schichtenlehre nach Schenker ist die Funktionstheorie oder auch eine funktional verstandene Stufentheorie dafür sehr wertvoll. Bedauerlich ist allerdings, dass sich die Funktionstheorie schon bei Riemann selbst, mehr noch in den Arbeiten eines Teils seiner Schüle ${ }^{20}$ und erst Recht in den prüfungsorientierten Curricula an Schulen und Hochschulen zu einer detailverliebten, gegenüber größeren Zusammenhänge aber ziemlich gleichgültigen Nomenklatur verselbständigt hat.

\section{Literatur}

Bernstein, David W. (2002), »Nineteenth-Century Harmonic Theory: The Austro-German Legacy", in: Christensen 2002, 788-794.

Christensen, Thomas (Hg.) (2002), The Cambridge History of Western Music Theory, Cambridge: Cambridge University Press.

19 Die potenzierende Verdichtung findet auf allen Ebenen statt: Das die Sequenz vermittelnde Kanonmotiv (es-as-as-g) wird ab Takt 8 in den Violinen einer weiteren Verkürzung unterzogen und mit halbtaktiger Einsatzfolge imitiert, wobei der letzte Einsatz in der Singstimme selbst zu den Textworten »wie erleuchtet» erklingt. Ein erneuter Anlauf des Motivs beginnt erst mit dem nächsten, überlappenden Abschnitt der Rede in H-Dur/Ces-Dur, wobei die Kleinterzstruktur der Sequenz auf die Großform übertragen ist.

20 Seidel 1966, 39. 
Gárdonyi, Zoltán (1969), „Neue Tonleiter- und Sequenztypen in Liszts Frühwerken«, Studia Musicologica Academiae Scientiarum Hungaricae 11, 169-199.

Gárdonyi, Zsolt / Hubert Nordhoff (2002), Harmonik, 2. Aufl., Wolfenbüttel: Möseler.

Haas, Bernhard (2004), Die neue Tonalität von Schubert bis Webern - Hören und Analysieren nach Albert Simon, Wilhelmshaven: Noetzel.

Hauptmann, Moritz (1853), Die Natur der Harmonik und der Metrik, Leipzig: Breitkopf \& Härtel.

Kurth, Ernst (1920), Romantische Harmonik und ihre Krise in Wagners 'Tristanı, Reprint der 3. Aufl. Berlin 1923, Hildesheim: Olms 1985.

Riemann, Hugo (1901a), »Musikalische Logik« [1872], in: ders., Präludien und Studien III, Leipzig: Breitkopf \& Härtel, Reprint Hildesheim: Olms 1967, 1-22.

— (1901b), „Zur Theorie der Konsonanz und Dissonanz«, in: ders., Präludien und Studien III, Leipzig: Breitkopf \& Härtel, Reprint Hildesheim: Olms 1967, 31-45.

— (1916), „Neue Beiträge zu einer Lehre von den Tonvorstellungen«, Jahrbuch der Musikbibliothek Peters 23, Leipzig: Peters, 1-21.

Rummenhöller, Peter (1966), »Moritz Hauptmann. Der Begründer einer transzendentaldialektischen Musiktheorie«, in: Vogel 1966, 11-38.

Schenker, Heinrich (1906), Neue Musikalische Theorien und Phantasien, Bd. 1: Harmonielehre, Stuttgart/Berlin: J. G. Cotta'sche Buchhandlung Nachfolger, Reprint Wien: Universal Edition 1978.

Schönberg, Arnold (1911), Harmonielehre, Wien: Universal Edition.

Schubert, Peter (2002), „Counterpoint Pedagogy in the Renaissance», in: Christensen 2002, 503-533.

Seidel, Elmar (1966), »Die Harmonielehre Hugo Riemanns«, in: Vogel 1966, 39-92.

Vogel, Martin (Hg.) (1966), Beiträge zur Musiktheorie des 19. Jahrhunderts, Regensburg: Bosse. 